\title{
Phase Stability of Rare-Earth Sesquioxides - New Approach
}

\author{
Giora Kimmel ${ }^{1 *}$ and Witold Lojkowski ${ }^{2}$ \\ ${ }^{1}$ Department of Materials Engineering, Ben-Gurion University of the Negev, Israel \\ ${ }^{2}$ Institute of High-Pressure Physics, Polish Academy of Sciences, Poland
}

*Corresponding author: Giora Kimmel, Department of Materials Engineering, BenGurion University of the Negev, Beer-Sheva, Israel.

Received Date: December 17, 2019

Published Date: January 03, 2020

\section{Opinion}

Based on low temperature synthesis it was stated that solid rare earth sesquioxides (RESOX) below $2000{ }^{\circ} \mathrm{C}$ belong to three structure types as function of temperature and metallic ionic radius. (I) Hexagonal for $\mathrm{La}_{2} \mathrm{O}_{3}$ and $\mathrm{Nd}_{2} \mathrm{O}_{3}$ with the highest metallic ionic radius, (II) Low Temperature (LT) cubic and High Temperature (HT) monoclinic for $\mathrm{Sm}_{2} \mathrm{O}_{3}, \mathrm{Eu}_{2} \mathrm{O}_{3}$ and $\mathrm{Gd}_{2} \mathrm{O}_{3}$ with medium metallic ionic radius, (III) cubic for $\mathrm{Dy}_{2} \mathrm{O}_{3}, \mathrm{Y}_{2} \mathrm{O}_{3}, \mathrm{Ho}_{2} \mathrm{O}_{3}, \mathrm{Er}_{2} \mathrm{O}_{3}, \mathrm{Tm}_{2} \mathrm{O}_{3}, \mathrm{Yb}_{2} \mathrm{O}_{3}$, $\mathrm{Lu}_{2} \mathrm{O}_{3}$ and $\mathrm{Sc}_{2} \mathrm{O}_{3}$ with low metallic ionic radius [1,2].

However, synthesis of the RESOX of (II) group by fast solidification yields stable monoclinic phases up to $2000{ }^{\circ} \mathrm{C}$. There are more unsolved dilemmas in the above model. (a) Why the monoclinic phase was never found when RESOX are formed by sol-gel technique, where nano-sized grains are formed? (b) Why the cubic phase was never found during solidification of oxides from (II) group? (c) Is in sol-gel products of (II) the transition occurring from LT cubic to HT monoclinic caused by grain growth or temperature factor? In order to resolve these dilemmas, we proposed an approach considering grain size.

Nowadays there is a wide agreement that the grain-size per volume parameter $(\Lambda)$ is a thermodynamic factor just like temperature, pressure and concentration. Navtrotsky [3] and Kimmel \& Zabicky [4] introduced the above parameter to explain why in ambient conditions nano materials' phases are stabilized by surface energy, while in bulk materials by the chemical energy. Phase transitions may occur during grain growth while crossing some critical $\Lambda$ the surface energy contribution becomes small and chemical energy takes control. This model is supported by experimental data of oxides based on $\mathrm{Ti}, \mathrm{Zr}, \mathrm{Al}, \mathrm{Hf}$, created using the sol-gel technique. In this technique it is easy to create at relatively low temperatures ceramic materials with particles in the nano scale. A common character of these oxides is that at low grain size crystallographic unit cell with lower $\Lambda$ is preferred. Consequently, in the nano scale there is preferences of crystals with isometric unit cells. A similar rule holds in bulk materials where isometric crystals are preferred at high temperature in order to reduce the free energy by increasing the entropy. How to apply the above theory to explain the above quoted transformations from LT cubic into HT higher density monoclinic phases for three lanthanide oxides $\mathrm{Gd}_{2} \mathrm{O}_{3}, \mathrm{Sm}_{2} \mathrm{O}_{3}$ and $\mathrm{Eu}_{2} \mathrm{O}_{3}$ - formed by sol-gel technique at room temperature?

In order to be able to distinguish between phase transitions driven by chemical energy and surface energy we designed experiments, where the $\Lambda$ value was approximately constant during temperature variation. This is important because in standard experiments with nanomaterials a temperature rise leads to grain growth. Therefore, phase transitions caused by grain growth can be wrongly interpreted as caused by a temperature increase. We found a way to slow down thermal grain growth of RESOX made by solgel technique. For that purpose, we synthesized mixtures of small amount of Samaria and gadolinia particles embedded in magnesia at room temperature. During firing at high temperatures, the small lanthanide oxides particle will be isolated from each other. This prevents grain growth by coalescence of particles. In this way we can separate two variables: temperature and grain size. There is no interaction between $\mathrm{MgO}$ and the lanthanide oxides [5]. However, pressure effects must be excluded. MgO has twice the larger linear expansion coefficient in comparison with the lanthanide oxides [6]. Thus, one must consider creation of hydrostatic pressure on small particles of lanthanide oxides embedded in $\mathrm{MgO}$ during cooling from high temperatures. We found that mixtures of $10 \%$ volume lanthanide oxides in $\mathrm{MgO}$ show a minor pressure effect. Cubic non isolated sol-gel products of Samaria and gadolinia become 
monoclinic at temperatures 1000 and $1300{ }^{\circ} \mathrm{C}$. In mixtures of $10 \%$ volume Samaria and gadolinia in $\mathrm{MgO}$ the cubic structure was retained in these temperatures. The meaning of these results is that without grain growth the transformation from cubic to monoclinic does not take place [7]. In this way it was proved that the cubic to monoclinic transition is not a thermal but a particle size effect. Consequently, the cubic phase is stabilized by small grain size due to smaller surface energy. For large grains the structure is not surface energy controlled but bulk-chemical energy controlled. The higher density monoclinic phase should be the stable phase for bulk materials at all temperatures for Samaria and gadolinia and probably also europia. The phase stability diagrams for two grain sizes are schematically shown in Figure 1.
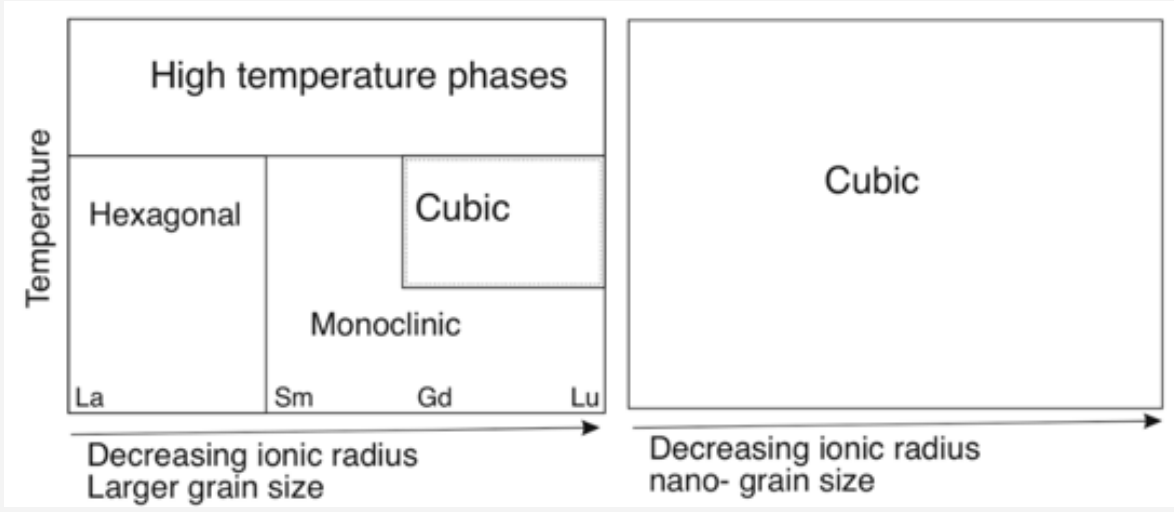

Figure 1: Schematic presentation of phase stability diagram for nano-sized REDOX and for conventional grain size [7].

Besides thermodynamic equilibrium, also kinetics plays an important role in phase structure formation. Rapid solidification at all temperatures formed monoclinic Samaria, europia and gadolinia. Moreover, the $\mathrm{m}=>\mathrm{c}$ transformation at high temperatures is a normal transition from low entropy monoclinic to high entropy cubic one. Fast solidification increases liquid under-cooling and leads to formation of smaller particles. Thus, fast cooling will form particles solidified in the LT monoclinic range, with small grains. Slow cooling will lead to larger grains created at higher temperatures, where the group (III) is in the HT cubic range. This model explains why large cubic particles and small monoclinic particles in micron scale were reported for yttria HT synthesis [8]. On the other hand, HT yttria products yielded very small cubic and the large monoclinic grains in the nanometric scale, exhibiting the size effect phenomenon [9].

Since the $m=>c$ transition has a negative slope versus the metal ion radius [10], in $\mathrm{Sc}_{2} \mathrm{O}_{3}$ the transition is in $0{ }^{\circ} \mathrm{C}$. Thus, at $\mathrm{RT} \mathrm{Sc}_{2} \mathrm{O}_{3}$ will always solidify as cubic.

\section{Acknowledgment}

None.

\section{Conflict of Interest}

No conflict of interest.

\section{References}

1. Roth RS, Schneider SJ (1960) Phase equilibria in systems involving the rare-earth oxides. part 1 . polymorphism of the oxides of the trivalent rare-earth ions. Journal of Research of the National Bureau of Standards-A Physics and Chemistry 64A(4): 309-316.

2. Warshaw I, Roy RJ (1961) Polymorphism of the rare earth sesquioxides. Phys Let 65: 2048-2051.

3. Navrotsky A (2003) Energetics of nanoparticle oxides: interplay between surface energy and polymorphism. Geochem Trans 4: 34-37.

4. Kimmel G, Zabicky J (2008) Stability, instability, metastability and grain size in nanocrystalline ceramic oxide systems. Solid State Phenomena 140: 29-36.

5. Lopato LM (1976) Highly refractory oxide systems containing oxides of rare-earth elements. Ceramugria International 2(1): 18-32.

6. Sato S, Takahomashi R, Kabune M, Gototh H (2009) Basic properties of rare earth oxides. Appl Catal A 356(1): 57-63.

7. Kimmel G, Shneck RZ, Lojkowski W, Porat Z, Chudoba T, et al. (2019) Phase stability of rare earth sesquioxides with grain size controlled in the nanoscale. J Am Cer Soc 102(7): 3829-3835.

8. Reeber R, Goessel K, Wang K (1995) Thermal expansion and molar volume of MgO, periclace, from 5 to $2900 \mathrm{~K}$. J Mineral 7(5): 1039-1047.

9. Guo B, Harvey A, Risbud SH, Kennedy IM (2006) The formation of cubic and monoclinic, $\mathrm{Y}_{2} \mathrm{O}_{3}$ nanoparticles in a gas-phase flame process. Phil Mag Let 86(7): 457-467.

10. Guo B, Luo ZP (2008) Particle size effect on the crystal structure of $\mathrm{Y}_{2} \mathrm{O}_{3}$ particles formed in a flame aerosol process. J Am Ceram Soc 91(5): 1653-1658. 